\title{
Neural Substrates for the Association Between Cigarette Smoking and Parkinson's Disease
}

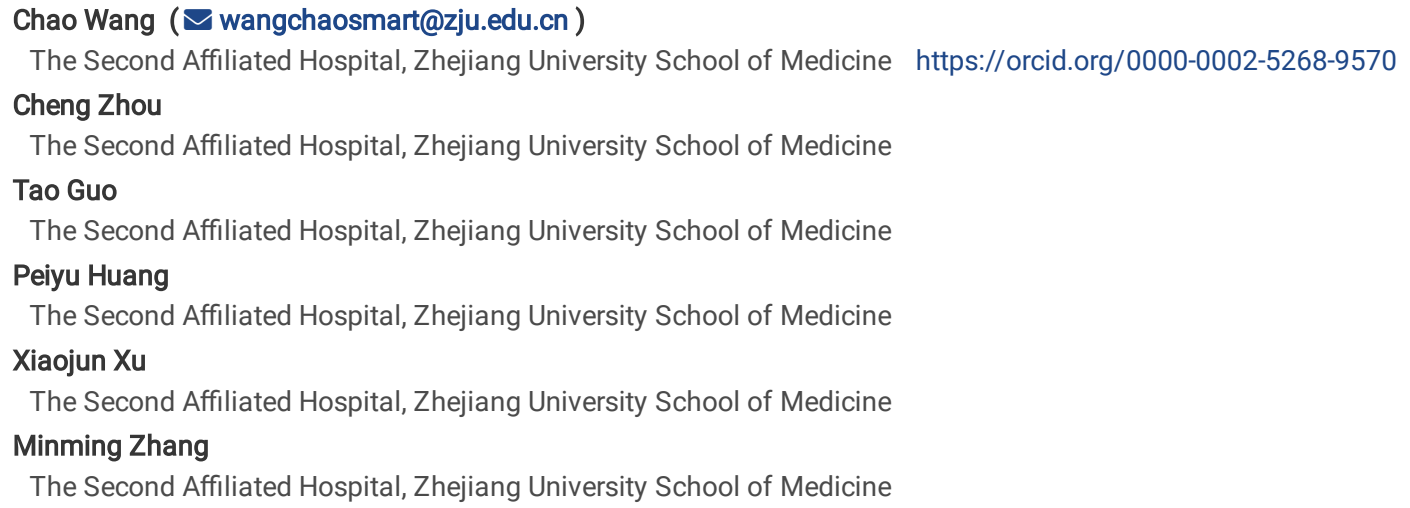




\section{Abstract}

Background: Mounting evidence revealed a causally protective effect of cigarette smoking on the risk of Parkinson's disease (PD). Meanwhile, cigarette smoking also was found to be associated with cognitive impairment in PD patients. However, the neural substrates of the protective and harmful effects of cigarette smoking on PD are not fully illustrated. Therefore, in this study, we explored the neural substrates for the protective and harmful effects of cigarette smoking on PD.

Methods: A total of 129 PD patients and 69 controls were recruited from the Parkinson's Progression Markers Initiative (PPMI) cohort, including 39 PD patients with regular smoking history (PD-S), 90 PD patients without regular smoking history (PD-NS), 26 healthy controls with regular smoking history (HC-S) and 43 healthy controls without regular smoking history (HC-NS). Striatal dopamine transporter (DAT) binding and gray matter (GM) volume of the whole brain were compared among the four groups.

Results: PD patients showed significantly reduced striatal DAT binding compared with healthy controls, and HC-S showed significantly reduced striatal DAT binding compared with HC-NS. Moreover, smoking and PD showed a significant interaction effect in the left medial prefrontal cortex (mPFC). PD-S showed reduced GM volume in the left mPFC compared with PD-NS.

Conclusions: This study is the first to reveal the neural substrates for both the protective and harmful effects of smoking on PD. The degeneration of dopaminergic neurons in PD results in a substantial reduction of the DAT and dopamine levels. Nicotine may act as a stimulant to inhibit the action of striatal DAT, increasing dopamine levels in the synaptic gap. The inverse alterations of dopamine levels between PD and nicotine addiction may be the neural substrates for the protective effect of smoking on the risk of PD. In addition, the mPFC atrophy in PD-S may be the neural substrates for the harmful impact of smoking on PD.

\section{Background}

Cigarette smoking is a leading cause of premature deaths, accounting for about 100 million deaths in the 20th century and an estimated 1 billion deaths in the 21 st century [1]. The association between cigarette smoking and a significantly increased risk of lung, heart, and vascular disease, as well as many cancers, is largely indisputable [2]. Parkinson's disease (PD) is the second-most common neurodegenerative disorder that affects $2-3 \%$ of elderly people >65 years old worldwide [3], which is characterized by bradykinesia, resting tremor, and muscular rigidity, and other non-motor symptoms. A meta-analysis of observational studies revealed the inverse association between cigarette smoking and the risk of PD [4]. Recently, a prospective study further demonstrated a causally protective effect of smoking on the risk of PD in a 65-year follow-up of 30,000 male British doctors [5]. However, the mechanism of the inverse association between smoking and PD is not fully illustrated.

PD is a progressive neurodegenerative disorder mainly caused by the degeneration of the dopaminergic neurons within the nigrostriatal pathway [6]. As a vital part of the nigrostriatal pathway, the striatum includes the caudate and putamen [7]. Dopamine transporter (DAT) imaging is an established diagnostic tool for degenerative parkinsonism. Reduced DAT binding is the result of a reduction in the number of neurons projecting from the substantia nigra to the striatum in PD $[8,9]$. The loss of dopamine neurons results in a substantial reduction of the DAT and dopamine levels [10]. Furthermore, DAT imaging can be helpful for the early diagnosis of PD [11] and also for distinguishing between parkinsonian syndromes with and without dopaminergic degeneration [12]. Chronic cigarette smoking results in a dysregulated reward processing, which is mediated by neuroadaptations in the mesolimbic dopamine system-particularly the striatum [13]. Nicotine is the primary addictive ingredient within tobacco, which acts as a presynaptic nicotinic acetylcholine receptors (nAChR) agonist, facilitates dopamine release, and makes cigarettes highly addictive [14]. Nicotine can boost extracellular dopamine levels by binding to nAChR in the striatum, and repeated nicotine stimulation may "hijack" natural reward circuits by increasing the drive to get nicotine [15]. Accumulating neuroimaging evidences have consistently shown cigarette smokers exhibited abnormal function in the striatum [16-19]. Boosted dopamine levels by nicotine effects of chronic smoking in healthy smokers may delay or even prevent the onset of PD. Here, we hypothesize that elevated dopamine levels in healthy smokers and reduced dopamine levels in PD may be the reason for the protective effect of smoking on the risk of PD. To indirectly prove this hypothesis, striatal DAT binding was used and compared among PD patients and healthy controls with or without regular smoking history using the Parkinson's Progress Markers Initiative (PPMI) cohort.

Meanwhile, several studies reported the harmful impact of smoking on PD. For example, smoking was associated with global cognitive impairment in PD patients, even those who had quit smoking [20]. Besides, smoking history showed an independent and dose-dependent association with impulse control disorders in PD patients [21]. Mounting evidence has demonstrated that cigarette smoking is linked with neurobiological and neurocognitive abnormalities [22]. Most neuroimaging studies examining the neurobiological consequences of smoking have focused on the volume of cortical and subcortical gray matter (GM) [23-25]. The most consistently reported brain areas of reduced GM volume include the prefrontal cortex (PFC), insula, thalamus, and cerebellum [24, 26, 27]. PFC is critical for cognitive control, which is heavily influenced by dopamine levels [28]. Here, we hypothesize that PD patients with regular smoking history show reduced GM volume in the PFC, which may be the neural substrates for the harmful impact of smoking on PD.

In the present study, therefore, to explore the neural substrates for the protective and harmful effects of smoking on PD, we compared striatal DAT binding and GM volume of the whole brain among the PD patients with regular smoking history (PD-S), PD patients without regular smoking history (PD-NS), healthy controls with regular smoking history (HC-S), and healthy controls without regular smoking history (HC-NS) using the PPMI cohort. Clarifying this issue might improve the understanding of the neurobiological substrates of the association between cigarette smoking and Parkinson's disease.

\section{Materials And Methods}

\section{Participants}


The data used in this study were all obtained from the PPMI database (www.ppmi-info.org) [29]. Data were downloaded on May 1, 2021. One hundred and thirty-two de novo PD patients and 70 control subjects with smoking history questionnaires were enrolled in the current study. One PD patient with poor quality of T1-weighted images, one PD patient with missing DAT scan, and one control subject with image format conversion error were excluded. The smoking history questionnaires included dozens of smoking questions range from "sqm1" to "smq9" (Supplemental Table). In this study, the subjects with "sqm2 = yes" were regarded as smoked subjects, while the subjects with "sqm2 = no" were regarded as non-smoked subjects. "sqm2" referred to the question "In your lifetime, have you ever regularly smoked cigarettes, that is, at least one cigarette per day for six months or longer?" One PD patient who didn't know/prefer not to answer the "smq2" was excluded. Finally, 129 PD patients (39 PD-S and 90 PD-NS patients) and 69 controls (26 HC-S and 43 HC-NS subjects) were included in this study (Figure 1).

In addition to the demographic variables (age, gender, education), the clinical variables were collected, including the Hoehn and Yahr (H-Y) stages, the Movement Disorders Society Unified Parkinson's Disease Rating Scale (MDS-UPDRS), Montreal Cognitive Assessment (MOCA), the Geriatric Depression Scale (GDS), the Scale for Outcomes for Parkinson's Disease-autonomic function (SCOPA-AUT), State and Trait Anxiety Scale (STAI), the Questionnaire for Impulsive-Compulsive Disorders in Parkinson's Disease (QUIP), the University of Pennsylvania Smell Identification Test (UPSIT), and the Epworth Sleepiness Scale (ESS).

\section{Dat Data Processing}

According to the PPMI imaging protocol, the DAT scan was acquired using single-photon emission computed tomography (SPECT) imaging. DAT imaging using $\left[{ }^{123}\right.$ I] FP -CIT SPECT was performed at PPMI imaging centers. Then, DAT images were sent to the Institute for Neurodegenerative Disorders for processing and calculation of striatal binding ratios (SBRs). For iterative reconstruction, SPECT raw projection data was imported to a HERMES (Hermes Medical Solutions, Skeppsbron 44, 11130 Stockholm, Sweden) system. Second, the reconstructed files were transferred to the PMOD (PMOD Technologies, Zurich, Switzerland) for subsequent processing. Third, attenuation correction and standard Gaussian 3D 6.0 mm filter was applied. Forth, these files were normalized to Montreal Neurologic Institute (MNI) space. Fifth, the highest striatal uptake of the trans-axial slice was identified, and the eight hottest striatal slices around it were averaged into generate a single slice image. Sixth, striatal regions of interest (ROIs) were then placed on the target regions (caudate and putamen) and reference region (occipital cortex). Finally, count densities for each ROI were extracted and used to calculate striatal binding ratios (SBRs) for each of the four striatal ROIs. SBRs were calculated as follows: SBRs = (target region/reference region)-1. Detailed DAT processing procedures can be found online (https://www.ppmiinfo.org/access-data-specimens/download-data/) and published documents [30, 31].

\section{Mri Data Acquisition And Preprocessing}

All baseline 3D T1-weighted imaging (T1WI) data were obtained from the PPMI database, which were acquired using Siemens $3.0 \mathrm{~T}$ scanners according to a standardized protocol. The scanning parameters were as follows: repetition time $(\mathrm{TR})=2300 \mathrm{~ms}$; echo time $(\mathrm{TE})=2.98 \mathrm{~ms}$; inversion time $=900 \mathrm{~ms}$; slice thickness $=1 \mathrm{~mm}$, field of view $=256 \mathrm{~mm}$, and matrix size $=240 \times 256$.

Before preprocessing, the MRI images of raw DICOM format were reviewed and converted into the Neuroimaging Informatics Technology Initiative (NII) format using MRICRON software. All NII images were preprocessed and analyzed using the CAT12 toolbox (Computational Anatomy Toolbox; http://dbm.neuro.unijena.de/cat/) implemented in SPM12 (http://www.fil.ion.ucl.ac. uk/spm/software/spm12/). CAT12 served as the platform for preprocessing the structural MRI data and offered a processing pipeline for voxel-based morphometry. For processing- and analysis-steps, pre-set parameters in accordance with standard protocol (http://www. neuro.uni-jena.de/cat12/CAT12-Manual.pdf) were used, applying default settings unless indicated otherwise. The procedure of data analysis was as follows:

1. T1 images are normalized to a template space and segmented into GM, white matter (WM), and cerebrospinal fluid (CSF).

2. Display slices for each subject to check the quality of spatial registration, including if the native volume had artifacts or if the native volumes had a wrong orientation. No subject had to be excluded due to poor quality.

3. Total intracranial volume (TIV) was estimated.

4. Mean correlation, weighted overall image quality, and Mahalanobis distance algorithms were used to quantify image quality after segmentation ("VBM data homogeneity" function). No subject had to be excluded due to poor quality.

5. Last, segmented GM images were smoothed with an $8 \mathrm{~mm}$ full-width-half-maximum (FWHM) isotropic Gaussian kernel.

6. For excluding artefacts on the grey/white matter border (i.e. incorrect voxel classification), an absolute grey matter threshold of 0.1 was applied to the VBM data.

7. In addition, the volume of each striatal nucleus (caudate and putamen) was estimated according to the Neuromorphometrics atlas and normalized by TIV for each subject (structure/ TIV*103).

\section{Statistical analysis}

Clinical and imaging characteristics analyses were performed using IBM SPSS Statistics 23 software (IBM Corporation, New York). The normality of the data was tested using Kolmogorov-Smirnov tests. Normally distributed data were assessed using parametric one-way analysis of variance (ANOVA) or $t$ test. Post hoc tests were performed after ANOVA. If the assumption of normality was not met, non-parametric Kruskal-Wallis $\mathrm{H}$ tests or Mann-Whitney $\mathrm{U}$ tests were used to assess between-group differences. Besides, chi-square tests were used to evaluate categorical variables. A Bonferroni correction was made to adjust for the number of comparisons of demographic and clinical characteristics. The corrected significance level is 0.05 divided by the total number of comparisons provided for that table-ie, $0.05 / 15=0.0033$ for clinical characteristics comparisons in table 1 and $0.05 / 15=0.0033$ for imaging characteristics comparisons in Table 2.

Table1. Demographic and clinical characteristics 


\begin{tabular}{|c|c|c|c|c|c|c|c|c|c|c|c|}
\hline & PD-S & PD-NS & HC-S & HC-NS & $\begin{array}{l}p \\
\text { values }\end{array}$ & & & & & & \\
\hline & $(n=39)$ & $(n=90)$ & $(n=26)$ & $(n=43)$ & $\begin{array}{l}4 \\
\text { Groups }\end{array}$ & $\begin{array}{l}\text { PD-S vs } \\
\text { PD-NS }\end{array}$ & $\begin{array}{l}\text { PD-S } \\
\text { vs HC- } \\
\text { S }\end{array}$ & $\begin{array}{l}\text { PD-S } \\
\text { vs HC- } \\
\text { NS }\end{array}$ & $\begin{array}{l}\text { PD- } \\
\text { NS vs } \\
\text { HC-S }\end{array}$ & $\begin{array}{l}\text { PD- } \\
\text { NS vs } \\
\text { HC-NS }\end{array}$ & $\begin{array}{l}\text { HC-S vs } \\
\text { HC-NS }\end{array}$ \\
\hline Age (years) & $61.5 \pm 10.1$ & $59.0 \pm 8.7$ & $59.1 \pm 10.5$ & $60.5 \pm 11.2$ & 0.545 & - & - & - & - & - & - \\
\hline $\begin{array}{l}\text { Gender (male/ } \\
\text { female) }\end{array}$ & $28 / 11$ & $56 / 34$ & $18 / 8$ & $28 / 15$ & 0.735 & - & - & - & - & - & - \\
\hline Education (years) & $16.2 \pm 2.1$ & $16.8 \pm 2.7$ & $16.5 \pm 2.6$ & $17.4 \pm 2.4$ & 0.149 & - & - & - & - & - & - \\
\hline $\begin{array}{l}\text { Regularly current } \\
\text { smokers }(n)\end{array}$ & 3 & 0 & 1 & 0 & $\mathrm{n} / \mathrm{a}$ & & & & & & \\
\hline $\mathrm{H}-\mathrm{Y}$ Stages & $1.4 \pm 0.6$ & $1.6 \pm 0.5$ & $\mathrm{n} / \mathrm{a}$ & $\mathrm{n} / \mathrm{a}$ & $\mathrm{n} / \mathrm{a}$ & 0.175 & - & - & - & - & - \\
\hline MDS-UPDRS Part I & $4.7 \pm 3.3$ & $4.8 \pm 3.3$ & $2.9 \pm 2.6$ & $3.1 \pm 2.5$ & $0.003^{*}$ & 0.910 & $0.025^{\star}$ & $0.024^{*}$ & $0.005^{\star}$ & $0.004^{*}$ & 0.716 \\
\hline MDS-UPDRS Part II & $5.8 \pm 3.9$ & $5.6 \pm 4.2$ & $0.3 \pm 0.9$ & $0.3 \pm 0.7$ & $<0.001^{*}$ & 0.633 & $<0.001^{*}$ & $<0.001^{*}$ & $<0.001^{*}$ & $<0.001^{*}$ & 0.649 \\
\hline MDS-UPDRS Part III & $19.0 \pm 7.3$ & $20.0 \pm 7.8$ & $0.7 \pm 1.0$ & $0.9 \pm 1.4$ & $<0.001^{*}$ & 0.386 & $<0.001^{*}$ & $<0.001^{*}$ & $<0.001^{*}$ & $<0.001^{*}$ & 0.674 \\
\hline $\begin{array}{l}\text { MDS-UPDRS Total } \\
\text { Score }\end{array}$ & $29.4 \pm 11.4$ & $30.4 \pm 12.1$ & $3.9 \pm 3.5$ & $4.2 \pm 3.3$ & $<0.001^{*}$ & 0.670 & $<0.001^{*}$ & $<0.001^{*}$ & $<0.001^{*}$ & $<0.001^{\star}$ & 0.465 \\
\hline MOCA & $27.5 \pm 2.4$ & $27.5 \pm 1.8$ & $28.1 \pm 1.1$ & $28.1 \pm 1.1$ & 0.31 & - & - & - & - & - & - \\
\hline GDS & $1.9 \pm 1.2$ & $1.9 \pm 2.2$ & $1.1 \pm 1.4$ & $1.1 \pm 1.8$ & $0.001^{*}$ & 0.203 & $0.005^{\star}$ & $<0.001^{*}$ & 0.087 & $0.01^{*}$ & 0.685 \\
\hline SCOPA-AUT & $9.0 \pm 5.4$ & $7.7 \pm 4.8$ & $4.9 \pm 3.2$ & $5.6 \pm 2.8$ & $<0.001^{*}$ & 0.211 & $0.001^{*}$ & $0.002^{*}$ & $0.005^{*}$ & $0.01^{*}$ & 0.182 \\
\hline STAI & $58.2 \pm 15.0$ & $63.0 \pm 17.5$ & $54.5 \pm 12.1$ & $53.3 \pm 12.1$ & 0.004 & - & - & - & - & - & - \\
\hline QUIP & $0.4 \pm 0.6$ & $0.2 \pm 0.60$ & $0.6 \pm 1.1$ & $0.1 \pm 0.3$ & 0.004 & - & - & - & - & - & - \\
\hline UPSIT & $23.3 \pm 8.4$ & $23.3 \pm 7.6$ & $33.4 \pm 5.9$ & $35.1 \pm 4.2$ & $<0.001^{*}$ & 0.987 & $<0.001^{*}$ & $<0.001^{*}$ & $<0.001^{*}$ & $<0.001^{*}$ & 0.337 \\
\hline ESS & $5.3 \pm 2.7$ & $5.7 \pm 3.6$ & $5.7 \pm 2.9$ & $5.5 \pm 3.6$ & 0.954 & - & - & - & - & - & - \\
\hline
\end{tabular}

*Significance level for comparisons is $\mathrm{p}<0.0033$ (after Bonferroni correction). Fewer than five participants in each group missed any one assessment. PD-S, PD patients with regular smoking history; PD-NS, PD patients without regular smoking history; HC-S, healthy controls with regular smoking history; HC-NS, healthy controls without regular smoking history; H-Y stages, Hoehn \& Yahr stages; MDS-UPDRS, Movement Disorders Society Unified Parkinson's Disease Rating Scale; MOCA, Montreal Cognitive Assessment; GDS, Geriatric Depression Scale; SCOPA-AUT, Scale for Outcomes for Parkinson's Disease-autonomic function; STAI, State trait anxiety score; QUIP, Questionnaire for Impulsive-Compulsive Disorders; UPSIT, University of Pennsylvania Smell Identification Test; ESS, Epworth Sleepiness Scale Score. 
Table 2

Imaging characteristics

\begin{tabular}{|c|c|c|c|c|c|c|c|c|c|c|}
\hline & PD-S & PD-NS & HC-S & HC-NS & $\begin{array}{l}p \\
\text { values }\end{array}$ & & & & & \\
\hline & $(n=39)$ & $(n=90)$ & $(n=26)$ & $(n=43)$ & $\begin{array}{l}4 \\
\text { Groups }\end{array}$ & $\begin{array}{l}\text { PD-S } \\
\text { VS PD- } \\
\text { NS }\end{array}$ & $\begin{array}{l}\text { PD-S } \\
\text { vs HC- } \\
\text { S }\end{array}$ & $\begin{array}{l}\text { PD-S } \\
\text { vs HC- } \\
\text { NS }\end{array}$ & $\begin{array}{l}\text { PD-NS } \\
\text { vs HC- } \\
\text { S }\end{array}$ & $\begin{array}{l}\text { PD-NS } \\
\text { vs HC- } \\
\text { NS }\end{array}$ \\
\hline $\begin{array}{l}\text { Caudate_L } \\
\text { SBRs }\end{array}$ & $2.08 \pm 0.55$ & $2.07 \pm 0.57$ & $2.93 \pm 0.49$ & $3.26 \pm 0.77$ & $<0.001^{*}$ & 0.956 & $<0.001^{*}$ & $<0.001^{*}$ & $<0.001^{*}$ & $<0.001^{*}$ \\
\hline $\begin{array}{l}\text { Caudate_R } \\
\text { SBRs }\end{array}$ & $2.10 \pm 0.63$ & $2.03 \pm 0.54$ & $2.92 \pm 0.49$ & $3.24 \pm 0.75$ & $<0.001^{*}$ & 0.539 & $<0.001^{*}$ & $<0.001^{*}$ & $<0.001^{*}$ & $<0.001^{*}$ \\
\hline $\begin{array}{l}\text { Putamen_L } \\
\text { SBRs }\end{array}$ & $0.76 \pm 0.29$ & $0.83 \pm 0.35$ & $2.09 \pm 0.51$ & $2.40 \pm 0.65$ & $<0.001^{*}$ & 0.419 & $<0.001^{*}$ & $<0.001^{*}$ & $<0.001^{*}$ & $<0.001^{*}$ \\
\hline $\begin{array}{l}\text { Putamen_R } \\
\text { SBRs }\end{array}$ & $0.87 \pm 0.38$ & $0.83 \pm 0.31$ & $2.08 \pm 0.48$ & $2.36 \pm 0.69$ & $<0.001^{*}$ & 0.659 & $<0.001^{*}$ & $<0.001^{*}$ & $<0.001^{*}$ & $<0.001^{\star}$ \\
\hline TIV & $1525.33 \pm 148.30$ & $1535.29 \pm 167.98$ & $1505.83 \pm 149.68$ & $1507.83 \pm 151.58$ & 0.742 & - & - & - & - & - \\
\hline $\begin{array}{l}\text { mPFC } \\
\text { Volume }\end{array}$ & $0.356 \pm 0.061$ & $0.382 \pm 0.050$ & $0.401 \pm 0.050$ & $0.360 \pm 0.055$ & $0.001^{*}$ & $0.012^{*}$ & $0.001^{*}$ & 0.748 & 0.116 & $0.026^{*}$ \\
\hline $\begin{array}{l}\text { Normalized } \\
\text { mPFC } \\
\text { Volume }\end{array}$ & $0.234 \pm 0.035$ & $0.250 \pm 0.025$ & $0.270 \pm 0.025$ & $0.240 \pm 0.033$ & $<0.001^{*}$ & $0.005^{\star}$ & $<0.001^{\star}$ & 0.382 & $0.008^{*}$ & $0.064^{*}$ \\
\hline $\begin{array}{l}\text { Caudate_L } \\
\text { Volume }\end{array}$ & $2.51 \pm 0.41$ & $2.57 \pm 0.37$ & $2.53 \pm 0.29$ & $2.44 \pm 0.36$ & 0.278 & - & - & - & - & - \\
\hline $\begin{array}{l}\text { Caudate_R } \\
\text { Volume }\end{array}$ & $2.58 \pm 0.44$ & $2.67 \pm 0.45$ & $2.64 \pm 0.32$ & $2.52 \pm 0.43$ & 0.248 & - & - & - & - & - \\
\hline $\begin{array}{l}\text { Putamen_L } \\
\text { Volume }\end{array}$ & $3.15 \pm 0.49$ & $3.26 \pm 0.52$ & $3.16 \pm 0.48$ & $3.21 \pm 0.55$ & 0.683 & - & - & - & - & - \\
\hline $\begin{array}{l}\text { Putamen_R } \\
\text { Volume }\end{array}$ & $3.00 \pm 0.38$ & $3.13 \pm 0.45$ & $3.07 \pm 0.43$ & $3.10 \pm 0.49$ & 0.547 & - & - & - & - & - \\
\hline $\begin{array}{l}\text { Normalized } \\
\text { Caudate_L } \\
\text { Volume }\end{array}$ & $1.64 \pm 0.23$ & $1.69 \pm 0.28$ & $1.69 \pm 0.19$ & $1.63 \pm 0.24$ & 0.799 & - & - & - & - & - \\
\hline $\begin{array}{l}\text { Normalized } \\
\text { Caudate_R } \\
\text { Volume }\end{array}$ & $1.70 \pm 0.25$ & $1.76 \pm 0.32$ & $1.76 \pm 0.21$ & $1.68 \pm 0.29$ & 0.640 & - & - & - & - & - \\
\hline $\begin{array}{l}\text { Normalized } \\
\text { Putamen_L } \\
\text { Volume }\end{array}$ & $2.07 \pm 0.31$ & $2.14 \pm 0.36$ & $2.12 \pm 0.37$ & $2.14 \pm 0.38$ & 0.795 & - & - & - & - & - \\
\hline $\begin{array}{l}\text { Normalized } \\
\text { Putamen_R } \\
\text { Volume }\end{array}$ & $1.98 \pm 0.24$ & $2.06 \pm 0.34$ & $2.06 \pm 0.36$ & $2.07 \pm 0.34$ & 0.536 & - & - & - & - & - \\
\hline
\end{tabular}

*Significance level for comparisons is $\mathrm{p}<0.0033$ (after Bonferroni correction). PD-S, PD patients with regular smoking history; PD-NS, PD patients without regi smoking history; HC-S, healthy controls with regular smoking history; HC-NS, healthy controls without regular smoking history; TIV, Total intracranial volume; I right; SBRs, striatal binding ratios.

Statistical analyses of imaging data were performed using the CAT12/SPM12 statistical module. The interaction effects of GM volume alteration between cigarette smoking and Parkinson's disease were determined using a full-factorial model with $2 \times 2$ ANOVA. TIV was included as a covariate to remove variance related to this global parameter of brain morphometry. To correct for multiple comparisons, we employed cluster-level family-wise error (FWE) correction at voxel level with $p<0.001$ and then corrected at the cluster level with non-stationary cluster extent correction. The significantly different brain region in the ANOVA analysis was saved as a mask using xjview (https://www.alivelearn.net/xjview/). Then, this mask was applied to extract the GM volume of every subject using the ROI Signal Extractor tool of Data Processing \& Analysis for Brain Imaging (DPABI) [32] for post hoc tests.

\section{Results}

In this study, a total of 129 PD patients (39 PD-S and 90 PD-NS) and 69 controls (26 HC-S and 43 HC-NS) were included. Baseline demographics and clinical variables were summarized in Table 1. There were no significant differences among PD-S, PD-NS, HC-S, and HC-NS in age, gender, and education. No difference was observed in the H-Y stages between the PD-S and PD-NS. Furthermore, MDS-UPDRS (specifically the part I, part II, part III, and total score), MOCA, GDS, SCOPA-AUT, STAI, QUIP, UPSIT, and ESS were also compared. Apart from MOCA, STAI, QUIP, and ESS, the clinical variables of MDS-UPDRS (part I, part II, part III, and total score), GDS, SCOPA-AUT, and UPSIT were different in PD subgroups compared with controls subgroups.

Striatal imaging variables were summarized in Table 2. One-way ANOVA showed significant differences of SBRs in both left and right striatal nuclei (caudate and putamen) among PD-S, PD-NS, HC-S, and HC-NS ( $<<0.001)$. Specifically, post hoc tests showed PD patients (PD-S and PD-NS) had lower SBRs than 
controls (HC-S and HC-NS). Moreover, HC-S showed lower SBRs than HC-NS in these four striatal nuclei (Figure 2). In addition, the volume of each striatal nucleus (caudate and putamen) was estimated. However, none of the striatal GM volume was different in cohorts of the four groups.

In addition, the interaction effects of GM volume alteration between cigarette smoking and Parkinson's disease were determined with $2 \times 2$ ANOVA. A significant interaction effect was detected in the left medial prefrontal cortex (MPFC) (Figure 3). Then, post hoc tests revealed that PD-S showed decreased GM volume in the left mPFC compared with PD-NS and HC-S.

\section{Discussion}

To our knowledge, the present study is the first to explore the neural substrates for the association between cigarette smoking and PD by analyzing both DAT and GM volume. The DAT results showed that PD patients had lower striatal DAT binding than controls, and HC-S had lower striatal DAT binding than HC-NS in both left and right striatal nuclei (caudate and putamen). In addition, a significant interaction effect between smoking and PD was detected in the left mPFC. Compared with PD-NS and HC-S, PD-S showed decreased GM volume in the left mPFC.

PD is a progressive neurodegenerative disorder characterized by selective degeneration of dopaminergic neurons in the substantia nigra pars compacta. Intraneuronal inclusions made up of a-synuclein aggregates, called Lewy bodies, are the neuropathological hallmark of PD [33]. DAT is a transmembrane sodium chloride dependent protein expressed only in presynaptic dopaminergic cells. DAT is responsible for the reuptake of free dopamine from the synaptic cleft back into the axonal button, and it plays a vital role in the spatial and temporal buffering of released dopamine levels [10]. The loss of dopamine neurons in the substantia nigra, first in the lateral layer and then in the medial region, is extensive and characteristic for PD, and it results in a substantial reduction of the DAT and dopamine levels [34]. In the current study, PD patients showed significantly decreased striatal DAT binding compared to healthy controls, which is in line with the previous studies [35]. However, the influence of smoking on striatal DAT binding of PD patients (PD-S vs PD-NS) was not detected. One explanation may be that there is a statistical 'floor' effect [36] that the DAT damage is severe enough in PD regardless of smoking history.

In this study, we also found that HC-S had significantly decreased striatal DAT binding in both left and right striatal nuclei (caudate and putamen) relative to HC-NS. A recent meta-analysis included seven previous small-sample studies of DAT imaging in smokers. It demonstrated a significant reduction in DAT availability in the current smokers compared to nonsmokers [37]. Further, the current study demonstrated a significant decrease in DAT availability in healthy individuals with regular smoking history ( 25 ex-smokers and only 1 current smoker, table 1 ) relative to 43 healthy nonsmokers. DAT mediates the reuptake of free dopamine from the synaptic cleft back into the axonal button [38]. Nicotine stimulates dopamine release, which has been demonstrated in non-human primates [39] and human [40]. The stimulants may act as the "DAT-blockers" to increase dopamine concentration in the synaptic gap and inhibit the action of DAT. This is supported by a DAT imaging study that nicotine may act as a stimulant on striatal DAT to reduce primarily elevated DAT density in adults with attention deficit hyperactivity disorder (ADHD) [41]. Furthermore, cigarette smoking has been demonstrated to inhibit monoamine oxidase (MAO) activity [42, 43]. MAO is a metabolic enzyme to breakdown dopamine. Thus, inhibited MAO is likely to result in greater dopamine concentration. Therefore, we believe that cigarettes may act as a stimulant to impact striatal DAT, increasing dopamine concentration in the synaptic gap and inhibiting the action of DAT. The present study demonstrated that chronic smoking decreased DAT availability in healthy smokers using [ [ ${ }^{123}$ I] FP-CIT SPECT. [ [ ${ }^{123}$ I] FP -CIT uptake may compete with intrinsic boosted dopamine levels by nicotine effects of chronic smoking and result in decreased DAT availability in healthy smokers. Therefore, we do not suggest that decreased DAT availability is caused by nigrostriatal degeneration in healthy smokers. We suggest that boosted dopamine levels by nicotine effects in healthy smokers may delay or even prevent the onset of PD. The reduction of striatal DAT binding was detected in both PD and healthy smokers in the current study. However, the different neurobiological mechanisms between PD and nicotine addiction result in inverse dopamine levels in PD and healthy smokers. This inverse alteration of dopamine levels in PD and healthy smokers may provide the reason for the protective effect of chronic smoking on PD that smokers exhibit a lower risk of PD relative to nonsmokers $[4,5]$. In the future, more studies are needed to confirm this hypothesis that chronic smoking affects striatal dopamine, DAT, and MAO.

In addition, we found smoking and PD showed a significant interaction effect in the left MPFC. PD-S showed decreased GM volume of mPFC relative to PD-NS and HC-S. Nicotine, the addictive component of cigarettes, activates nAChR in the VTA, resulting in dopamine release in the mesolimbic area, the corpus striatum, and the frontal cortex. Dopamine release results in a pleasurable experience, which is critical for the reinforcing effects (effects that promote selfadministration) of nicotine [44]. The mPFC plays a crucial role in addictive behavior. A subset of the mPFC neurons forms neuronal ensembles to encode the coupling between the reward of drug use and the associated contexts. And reactivation of the neuronal ensembles caused by drug-associated contexts during abstinence can provoke drug relapse [45]. The mPFC contributes to goal-directed behavior in response to motivational salience and reward expectation [46]. Functional MRI studies have shown that decreased reward-related MPFC activity is related to impaired motivation and poor self-control in individuals with addictions [47]. In addition, increased reward-related mPFC activity is related to reduced smoking in smokers [48].

The mPFC is an essential component of the cortex-limbic-striatal circuit, which has been proved to be related to the pathophysiology of non-motor symptoms of PD.[49] In addition to motor dysfunction, dopaminergic degeneration is also associated with cognitive deficits in PD [50]. Both PD patients and mice with ventral tegmental area (VTA) dopamine depletion can lead to attenuated delta activity (1-4 Hz) in the mPFC during interval timing [51]. Interval timing is a task of estimating an interval of several seconds as guided by a cue and requires executive resources such as working memory and attention to time [51].

Furthermore, optogenetic stimulation of the mPFC neurons expressing D1 dopamine receptors at delta frequencies can compensate for the impaired temporal control of action caused by VTA dopamine depletion [51]. The rodents with 6-hydroxydopamine (6-OHDA) induced lesion in the medial forebrain successfully recapitulates PD motor impairment and several non-motor symptoms [52]. Total smoking pack-years has been found to be associated with global cognitive impairment in PD patients with smoking history even in patients who have quit smoking [20]. In the current study, PD-S showed reduced GM volume of mPFC compared to PD-NS and HC-S. Taken together, these findings suggest that reduced GM volume of mPFC may be the neural substrates for the harmful impact of chronic smoking on PD that chronic smoking was associated with cognitive impairment in PD patients [20]. 
The current study has several limitations. In this retrospective analysis, more detailed smoking behavior characteristics on smoking history were not collected, such as years smoked, cigarettes smoked per day, smoking initiation age, and nicotine dependence levels. Thus, further association analysis can not be performed between altered imaging biomarkers and smoking behavior characteristics. Further, this retrospective study design does not allow us to determine causality; we can not determine whether the alterations of DAT binding and GM anatomy predispose to smoking initiation or whether chronic smoking influences DAT binding and GM anatomy. This question could be answered by prospective longitudinal studies. Future longitudinal evaluations are needed to explore the alterations of DAT binding and GM anatomy before and after the initiation of smoking.

\section{Conclusion}

This study is the first to comprehensively reveal the neural substrates for both the protective and harmful effects of smoking on PD. In this study, we reported baseline clinical and imaging characteristics of PD patients and healthy controls with or without regular smoking history. PD patients showed significantly reduced striatal DAT binding compared with healthy controls. PD is characterized by selective degeneration of dopaminergic neurons, and it results in a substantial reduction of the DAT and dopamine levels. Interestingly, HC-S showed significantly reduced striatal DAT binding compared with HC-NS. Nicotine may act as a stimulant to inhibit the action of striatal DAT, increasing dopamine levels in the synaptic gap. Despite the reduction of striatal DAT binding in both PD and healthy smokers, the inverse alteration of dopamine levels due to different neurobiological mechanisms between PD and nicotine addiction may be the neural substrates for the protective effect of smoking on the risk of PD. In addition, smoking and PD showed a significant interaction effect in the left mPFC. The mPFC atrophy in PD-S may be the neural substrates for the harmful impact of smoking on PD that chronic smoking was associated with cognitive impairment in PD.

\section{Abbreviations}

ANOVA: one-way analysis of variance; CSF: cerebrospinal fluid; DAT: dopamine transporter binding; DPABI: Data Processing \& Analysis for Brain Imaging; ESS: Epworth Sleepiness Scale; FEW: family-wise error; FWHM: full-width-half-maximum; GDS: Geriatric Depression Scale; GM: gray matter; HC-S: healthy controls with regular smoking history; HC-NS: healthy controls without regular smoking history; H-Y: Hoehn and Yahr; MDS-UPDRS: Movement Disorders Society Unified Parkinson's Disease Rating Scale; MNI: Montreal Neurologic Institute; MOCA: Montreal Cognitive Assessment; mPFC: medial prefrontal cortex; nAChR: nicotinic acetylcholine receptors; NII: Neuroimaging Informatics Technology Initiative; PD: Parkinson's disease; PPMI: Parkinson's Progression Markers Initiative; PD-S: Parkinson's disease patients with regular smoking history; PFC: prefrontal cortex; PD-NS: Parkinson's disease patients without regular smoking history; QUIP: Questionnaire for Impulsive-Compulsive Disorders in Parkinson's Disease; SBRs: striatal binding ratios; ROIs: regions of interest; SCOPA-AUT: Scale for Outcomes for Parkinson's Disease-autonomic function; SPECT: single-photon emission computed tomography; STAI: State and Trait Anxiety Scale; T1WI: T1-weighted imaging; TE: echo time; TIV: Total intracranial volume; TR: repetition time; UPSIT: University of Pennsylvania Smell Identification Test; WM: white matter.

\section{Declarations}

\section{Acknowledgments}

Data used in the preparation of this article were obtained from the PPMI database (www.ppmi-info.org/data). For up-to-date information on the study, visit www.ppmi-info.org. PPMI, a public private partnership, is funded by The Michael J. Fox Foundation for Parkinson's Research and funding partners, including AbbVie, Allergan, Avid, Biogen, Bristol-Myers Squibb, Covance, GE Healthcare, Genentech, GlaxoSmithKline, Lilly, Lundbeck, Merck, Meso Scale Discovery, Pfizer, Piramal, Roche, SANOFI GENZYME, Servier, Takeda, Teva, UCB, and GolubCapital.

\section{Authors' contributions}

C.W. and M.Z. conceived the study. C.W., C.Z., and T.G. collected data. C.W., C.Z., T.G., P.H., and X.X. performed statistical analyses. C.W. drafted the manuscript. All authors contributed to critical revision of the manuscript.

\section{Funding}

This work was supported by Zhejiang Provincial Natural Science Foundation of China under Grant No. LY21H180003, and the 13th Five-year Plan for National Key Research and Development Program of China under Grant No. 2016YFC1306600.

\section{Availability of data and materials}

All the data supporting the conclusions of the current study are presented in the figures and they are available from the corresponding authors upon reasonable request. There are no restrictions on data availability.

\section{Ethics approval and consent to participate}

The data included in this study were from the PPMI database. The PPMI study was approved by the Institutional Review Board or Independent Ethics Committee of all participating sites in Europe, including Attikon University Hospital (Greece), Hospital Clinic de Barcelona and Hospital Universitario Donostia (Spain), Innsbruck University (Austria), Paracelsus-Elena-Klinic Kassel/University of Marburg (Germany), Imperial College London (UK), Pitié-Salpêtrière Hospital (France), University of Salerno (Italy), and in the USA, including Emory University, Johns Hopkins University, University of Alabama at Birmingham, PD and Movement Disorders Center of Boca Raton, Boston University, Northwestern University, University of Cincinnati, Cleveland Clinic Foundation, Baylor College of Medicine, Institute for Neurodegenerative Disorders, Columbia University Medical Center, Beth Israel Medical Center, University of Pennsylvania, 
Oregon Health and Science University, University of Rochester, University of California at San Diego, and University of California, San Francisco. Informed consent was provided according to the Declaration of Helsinki.

\section{Consent for publication}

All authors have reviewed the final manuscript and consent to publication.

\section{Competing interests}

The authors declare that they have no competing interests.

\section{Author details}

${ }^{1}$ Department of Radiology, The Second Affiliated Hospital, Zhejiang University School of Medicine, Hangzhou, China

\section{References}

1. Pirie K, Peto R, Reeves GK, Green J, Beral V: The 21 st century hazards of smoking and benefits of stopping: a prospective study of one million women in the UK. Lancet 2013, 381:133-141.

2. Doll R, Peto R, Boreham J, Sutherland I: Mortality in relation to smoking: 50 years' observations on male British doctors. Bmj 2004, 328:1519.

3. Poewe W, Seppi K, Tanner CM, Halliday GM, Brundin P, Volkmann J, Schrag AE, Lang AE: Parkinson disease. Nat Rev Dis Primers 2017, 3:17013.

4. Li X, Li W, Liu G, Shen X, Tang Y: Association between cigarette smoking and Parkinson's disease: A meta-analysis. Arch Gerontol Geriatr 2015, 61:510516.

5. Mappin-Kasirer B, Pan H, Lewington S, Kizza J, Gray R, Clarke R, Peto R: Tobacco smoking and the risk of Parkinson disease: A 65-year follow-up of 30,000 male British doctors. Neurology 2020, 94:e2132-e2138.

6. Shimohama S, Sawada H, Kitamura Y, Taniguchi T: Disease model: Parkinson's disease. Trends Mol Med 2003, 9:360-365.

7. Whetsell WO, Jr.: The mammalian striatum and neurotoxic injury. Brain Pathol 2002, 12:482-487.

8. Kish SJ, Shannak K, Hornykiewicz O: Uneven pattern of dopamine loss in the striatum of patients with idiopathic Parkinson's disease. Pathophysiologic and clinical implications. N Engl J Med 1988, 318:876-880.

9. Fearnley JM, Lees AJ: Ageing and Parkinson's disease: substantia nigra regional selectivity. Brain 1991, 114 (Pt 5):2283-2301.

10. Uhl GR: Dopamine transporter: basic science and human variation of a key molecule for dopaminergic function, locomotion, and parkinsonism. Mov Disord 2003, 18 Suppl 7:S71-80.

11. Jennings D, Siderowf A, Stern M, Seibyl J, Eberly S, Oakes D, Marek K: Imaging prodromal Parkinson disease: the Parkinson Associated Risk Syndrome Study. Neurology 2014, 83:1739-1746.

12. Perju-Dumbrava LD, Kovacs GG, Pirker S, Jellinger K, Hoffmann M, Asenbaum S, Pirker W: Dopamine transporter imaging in autopsy-confirmed Parkinson's disease and multiple system atrophy. Mov Disord 2012, 27:65-71.

13. Wise RA: Roles for nigrostriatal-not just mesocorticolimbic--dopamine in reward and addiction. Trends Neurosci 2009, 32:517-524.

14. Wonnacott S: Presynaptic nicotinic ACh receptors. Trends Neurosci 1997, 20:92-98.

15. Volkow ND, Fowler JS, Wang GJ, Baler R, Telang F: Imaging dopamine's role in drug abuse and addiction. Neuropharmacology 2009 , 56 Suppl 1:3-8.

16. Bühler M, Vollstädt-Klein S, Kobiella A, Budde H, Reed LJ, Braus DF, Büchel C, Smolka MN: Nicotine dependence is characterized by disordered reward processing in a network driving motivation. Biol Psychiatry 2010, 67:745-752.

17. David SP, Munafò MR, Johansen-Berg H, Smith SM, Rogers RD, Matthews PM, Walton RT: Ventral striatum/nucleus accumbens activation to smokingrelated pictorial cues in smokers and nonsmokers: a functional magnetic resonance imaging study. Biol Psychiatry 2005, 58:488-494.

18. Sweitzer MM, Geier CF, Joel DL, McGurrin P, Denlinger RL, Forbes EE, Donny EC: Dissociated effects of anticipating smoking versus monetary reward in the caudate as a function of smoking abstinence. Biol Psychiatry 2014, 76:681-688.

19. Wang C, Huang P, Shen Z, Qian W, Wang S, Jiaerken Y, Luo X, Li K, Zeng Q, Zhou C, et al: Increased striatal functional connectivity is associated with improved smoking cessation outcomes: A preliminary study. Addict Biol 2021, 26:e12919.

20. Doiron M, Dupré N, Langlois M, Provencher P, Simard M: Smoking history is associated to cognitive impairment in Parkinson's disease. Aging Ment Health 2017, 21:322-326.

21. Valença GT, Glass PG, Negreiros NN, Duarte MB, Ventura LM, Mueller M, Oliveira-Filho J: Past smoking and current dopamine agonist use show an independent and dose-dependent association with impulse control disorders in Parkinson's disease. Parkinsonism Relat Disord 2013, 19:698-700.

22. Durazzo TC, Meyerhoff DJ, Nixon SJ: Chronic cigarette smoking: implications for neurocognition and brain neurobiology. Int J Environ Res Public Health 2010, 7:3760-3791.

23. Yang Z, Zhang Y, Cheng J, Zheng R: Meta-analysis of brain gray matter changes in chronic smokers. Eur J Radio/ 2020, 132:109300.

24. Sutherland MT, Riedel MC, Flannery JS, Yanes JA, Fox PT, Stein EA, Laird AR: Chronic cigarette smoking is linked with structural alterations in brain regions showing acute nicotinic drug-induced functional modulations. Behav Brain Funct 2016, 12:16

25. Fritz HC, Wittfeld K, Schmidt CO, Domin M, Grabe HJ, Hegenscheid K, Hosten N, Lotze M: Current smoking and reduced gray matter volume-a voxel-based morphometry study. Neuropsychopharmacology 2014, 39:2594-2600. 
26. Wang C, Xu X, Qian W, Shen Z, Zhang M: Altered human brain anatomy in chronic smokers: a review of magnetic resonance imaging studies. Neurol Sci 2015, 36:497-504.

27. Shen Z, Huang P, Wang C, Qian W, Yang Y, Zhang M: Cerebellar Gray Matter Reductions Associate With Decreased Functional Connectivity in NicotineDependent Individuals. Nicotine Tob Res 2018, 20:440-447.

28. Ott T, Nieder A: Dopamine and Cognitive Control in Prefrontal Cortex. Trends Cogn Sci 2019, 23:213-234.

29. The Parkinson Progression Marker Initiative (PPMI). Prog Neurobiol 2011, 95:629-635.

30. Tinaz S, Chow C, Kuo PH, Krupinski EA, Blumenfeld H, Louis ED, Zubal G: Semiquantitative Analysis of Dopamine Transporter Scans in Patients With Parkinson Disease. Clin Nucl Med 2018, 43:e1-e7.

31. Tagare HD, DeLorenzo C, Chelikani S, Saperstein L, Fulbright RK: Voxel-based logistic analysis of PPMI control and Parkinson's disease DaTscans. Neuroimage 2017, 152:299-311.

32. Yan CG, Wang XD, Zuo XN, Zang YF: DPABI: Data Processing \& Analysis for (Resting-State) Brain Imaging. Neuroinformatics 2016, 14:339-351.

33. Dickson DW: Neuropathology of Parkinson disease. Parkinsonism Relat Disord 2018, 46 Suppl 1:S30-s33.

34. Sulzer D: Multiple hit hypotheses for dopamine neuron loss in Parkinson's disease. Trends Neurosci 2007, 30:244-250.

35. Palermo G, Ceravolo R: Molecular Imaging of the Dopamine Transporter. Cells 2019, 8.

36. Bohnen NI, Kuwabara H, Constantine GM, Mathis CA, Moore RY: Grooved pegboard test as a biomarker of nigrostriatal denervation in Parkinson's disease. Neurosci Lett 2007, 424:185-189.

37. Ashok AH, Mizuno Y, Howes OD: Tobacco smoking and dopaminergic function in humans: a meta-analysis of molecular imaging studies. Psychopharmacology (Berl) 2019, 236:1119-1129.

38. Giros B, Caron MG: Molecular characterization of the dopamine transporter. Trends Pharmacol Sci 1993, 14:43-49.

39. Cumming P, Rosa-Neto P, Watanabe H, Smith D, Bender D, Clarke PB, Gjedde A: Effects of acute nicotine on hemodynamics and binding of [11C]raclopride to dopamine D2,3 receptors in pig brain. Neuroimage 2003, 19:1127-1136.

40. Brody AL, Mandelkern MA, Olmstead RE, Allen-Martinez Z, Scheibal D, Abrams AL, Costello MR, Farahi J, Saxena S, Monterosso J, London ED: Ventral striatal dopamine release in response to smoking a regular vs a denicotinized cigarette. Neuropsychopharmacology 2009, 34:282-289.

41. Krause KH, Dresel SH, Krause J, Kung HF, Tatsch K, Ackenheil M: Stimulant-like action of nicotine on striatal dopamine transporter in the brain of adults with attention deficit hyperactivity disorder. Int J Neuropsychopharmacol 2002, 5:111-113.

42. Lewis A, Miller JH, Lea RA: Monoamine oxidase and tobacco dependence. Neurotoxicology 2007, 28:182-195.

43. Berlin I, Anthenelli RM: Monoamine oxidases and tobacco smoking. Int J Neuropsychopharmacol 2001, 4:33-42.

44. Benowitz NL: Nicotine addiction. N Engl J Med 2010, 362:2295-2303.

45. Bossert JM, Stern AL, Theberge FR, Cifani C, Koya E, Hope BT, Shaham Y: Ventral medial prefrontal cortex neuronal ensembles mediate context-induced relapse to heroin. Nat Neurosci 2011, 14:420-422.

46. Goldstein RZ, Volkow ND: Drug addiction and its underlying neurobiological basis: neuroimaging evidence for the involvement of the frontal cortex. Am $J$ Psychiatry 2002, 159:1642-1652.

47. Goldstein RZ, Alia-Klein N, Tomasi D, Zhang L, Cottone LA, Maloney T, Telang F, Caparelli EC, Chang L, Ernst T, et al: Is decreased prefrontal cortical sensitivity to monetary reward associated with impaired motivation and self-control in cocaine addiction? Am J Psychiatry 2007, 164:43-51.

48. Garrison KA, Yip SW, Balodis IM, Carroll KM, Potenza MN, Krishnan-Sarin S: Reward-related frontostriatal activity and smoking behavior among adolescents in treatment for smoking cessation. Drug Alcohol Depend 2017, 177:268-276.

49. Ramos BP, Arnsten AF: Adrenergic pharmacology and cognition: focus on the prefrontal cortex. Pharmacol Ther 2007, 113:523-536.

50. Kaasinen V, Nurmi E, Brück A, Eskola O, Bergman J, Solin O, Rinne JO: Increased frontal [(18)F]fluorodopa uptake in early Parkinson's disease: sex differences in the prefrontal cortex. Brain 2001, 124:1125-1130.

51. Kim YC, Han SW, Alberico SL, Ruggiero RN, De Corte B, Chen KH, Narayanan NS: Optogenetic Stimulation of Frontal D1 Neurons Compensates for Impaired Temporal Control of Action in Dopamine-Depleted Mice. Curr Biol 2017, 27:39-47.

52. Marshall CA, King KM, Kortagere S: Limitations of the rat medial forebrain lesion model to study prefrontal cortex mediated cognitive tasks in Parkinson's disease. Brain Res 2019, 1702:105-113.

\section{Figures}




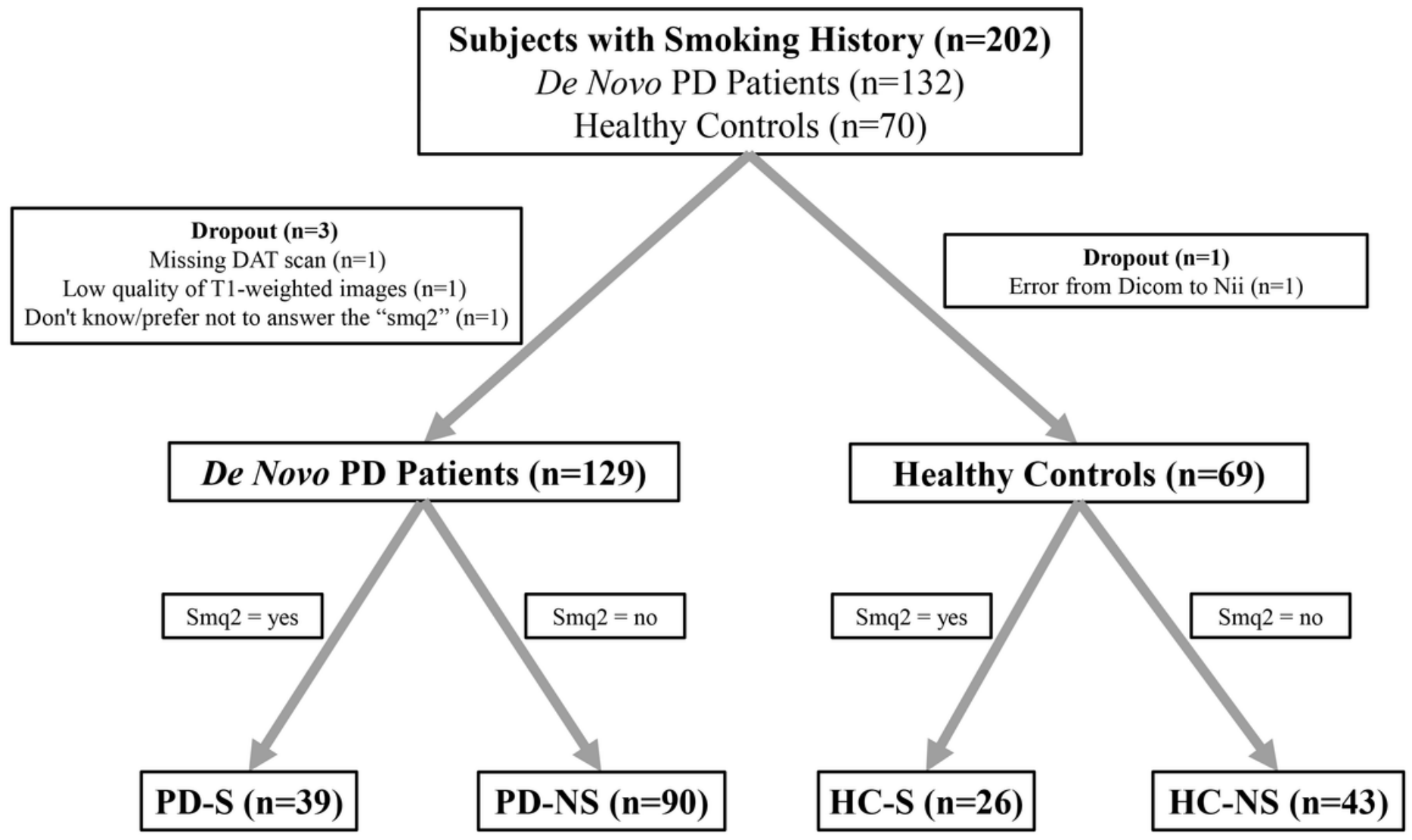

Figure 1

Flow diagram of the study population. 

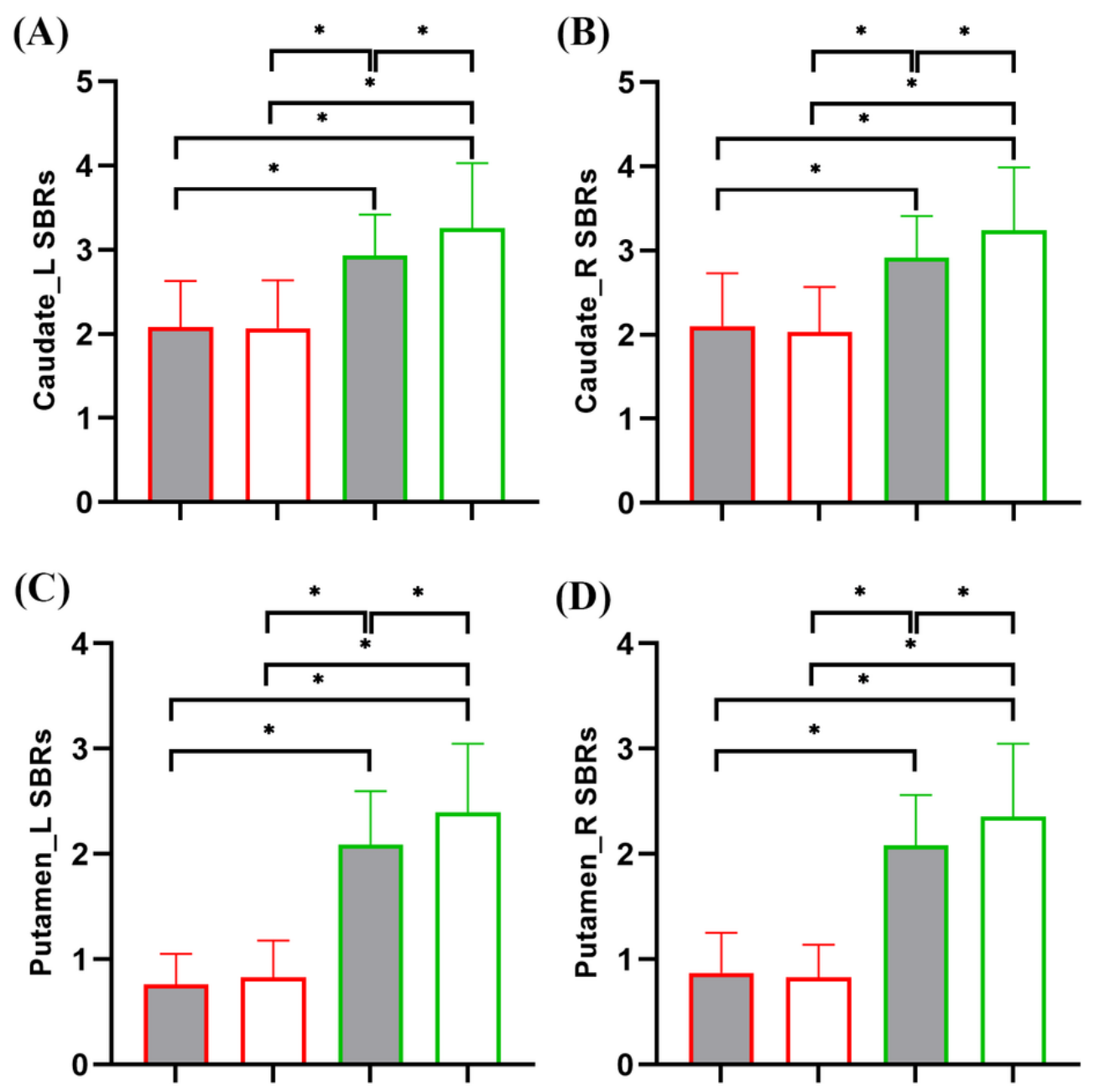

Figure 2

(A) PD patients (PD-S and PD-NS) showed lower SBRs than healthy controls (HC-S and HC-NS), and HC-S showed lower SBRs than HC-NS in each of the four striatal nuclei, including the left caudate (A), right caudate (B), left putamen (C) and right putamen (D). SBRs, striatal binding ratios.
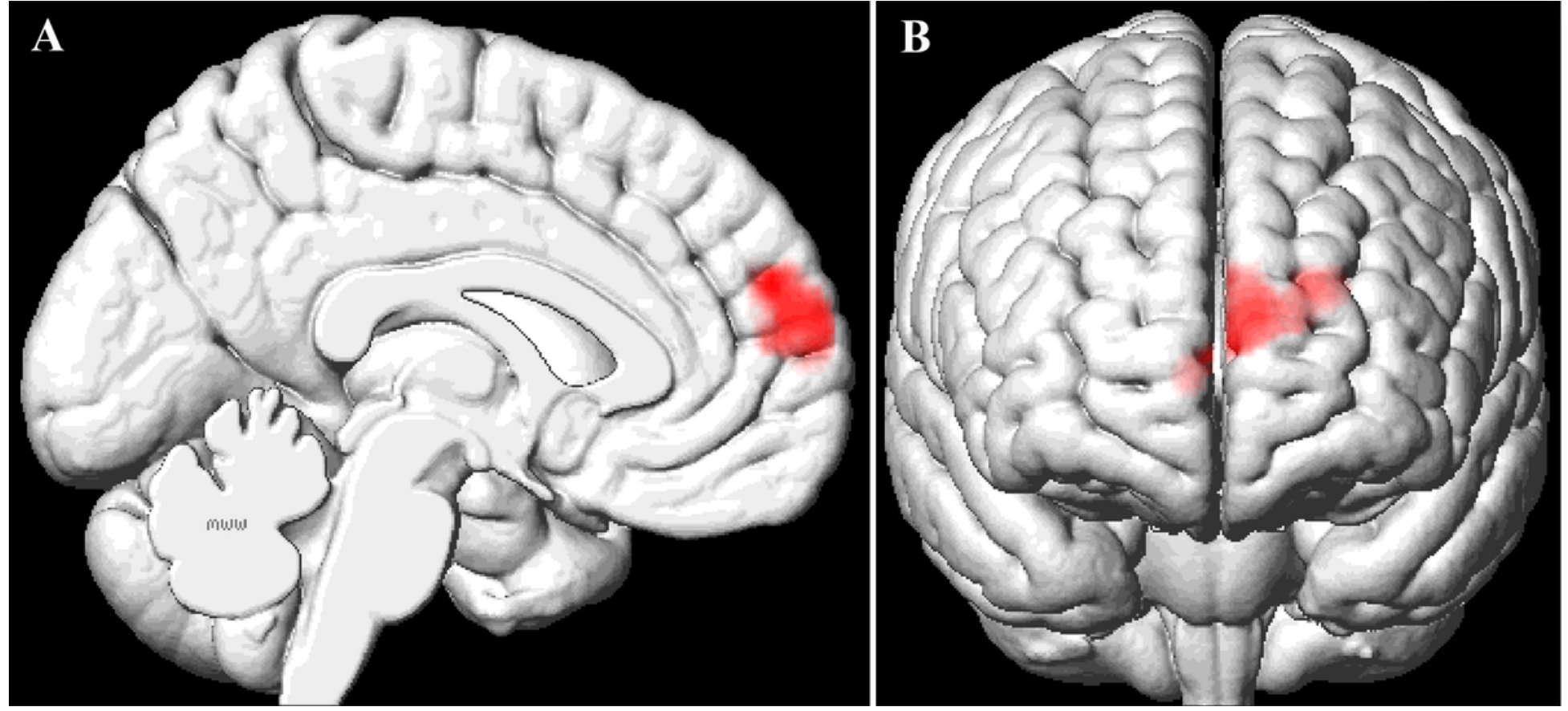
Using $2 \times 2$ analysis of variance (ANOVA), significant interaction effect was detected in the left mPFC (BA10) (medial frontal gyrus) (Peak MNI coordinate: -22.5, 57, 24; F values: 21.0359; Cluster size:1599). mPFC, medial prefrontal cortex.

\section{Supplementary Files}

This is a list of supplementary files associated with this preprint. Click to download.

- SupplementalTable.docx 